\title{
Effective strain range-based prediction and analysis of the fatigue life of pipette shaft seal ring
}

\author{
Chenxue Wang ${ }^{1, a}$, Qian $\mathrm{Su}^{2, \mathrm{a}}$, Zeng Huang ${ }^{2,}$, Zhiping $\mathrm{Lian}^{3}$, and $\mathrm{Yu} \mathrm{Liu}{ }^{4}$ \\ ${ }^{1}$ Graduate School of Science and Technology, Niigata University, Niigata 9502181, Japan \\ ${ }^{2}$ Department of Mechanical Engineering, Guangxi Technological College of Machinery and Electricity, Guangxi 530007, PR \\ China \\ ${ }^{3}$ Vocational Basic Education Department of Vocational Technical college, Guangxi Normal University, Guangxi 541004, PR \\ China \\ ${ }^{4}$ School of Mechanical, Materials, Mechatronic and Biomedical Engineering, University of Wollongong, Wollongong, NSW 2522, \\ Australia
}

Received: 4 June 2020 / Accepted: 1 July 2021

\begin{abstract}
The accuracy of nucleic acid extraction and diagnosis depends on the sealing performance of the pipetting device. The rubber seal ring at the front end of the pipette shaft is a critical component for ensuring the sealing performance of the pipetting device. In this study, an accurate prediction and analysis were made on the fatigue life of the pipette shaft seal ring. ABAQUS was used to simulate the assembly process of pipette shaft seal ring and disposable tip, and the dangerous cross-section segments on the seal rings were preliminarily identified according to a stress nephogram. Based on the continuum damage mechanics theory, the fatigue life prediction model was built where the effective strain was taken as a damage parameter, and the fatigue life of the dangerous cross-section of the pipette shaft seal ring was calculated. By observing failed seal rings in actual use, it was found that the worn positions of the failed seal rings were the same as the sites of the shortest-lived node segments, thus verifying the accuracy of fatigue life prediction model and fatigue life analysis.
\end{abstract}

Keywords: Pipette shaft seal ring / ABAQUS / effective strain range / fatigue life

\section{Introduction}

Pipettes and disposable tips have been indispensable for the detection and diagnosis of virus nucleic acid. The seal ring at the front end of a pipette shaft is a component critical to the sealing performance of the pipette. It works under special and complicated conditions. Figure 1 shows the process of disposable tip and pipette shaft assembly. It mainly comprises a pipette piston, a pipette shaft, an O-ring, and a disposable tip. In the process of a pipette and tip assembly, the automatic pipetting station inserts the pipette shaft vertically into the tip with the seal ring inserted about $3-4 \mathrm{~mm}$ deep into the tip. Owing to the installation of a seal ring at the front end of the shaft, after the insertion of the pipette shaft into the tip, the crosssection of the seal ring comes under contact and compression stresses, which brings about elastic deformation of the seal ring and produces a self-sealing effect,

\footnotetext{
${ }^{a}$ Chenxue Wang and Qian Su contributed equally to the work.

* e-mail: dguidance@163.com
}

thereby ensuring the excellent sealing performance of the pipette. After pipetting is completed, the tip is removed by pressing the tip push-off button and pushing down the push-off plate. Taking a testing laboratory, where there are 1000 samples awaiting testing, as an example, tens of thousands of pipetting operations need to be carried out there. As for a complete pipetting cycle, it comprises six steps, including the pipette tip, setting the volume, tip prewashing, depressing the Plunger, withdrawal of solution, expulsion of solution, and discarding the tip.

As a consequence, the seal ring at the front end of the pipette shaft has to be inserted into and pulled out of tips for tens of thousands of times. During frequent pipette-tip assemblies, the seal ring must bear a periodically fluctuating load from compression and shear stresses, i.e., it is always in a complex stress state, and its fatigue life has a direct bearing on the airtightness and reliability of the pipette. Therefore, it is quite necessary to predict the fatigue life of pipette shaft seal rings.

There are very few reports on fatigue life prediction of pipette shaft seal rings susceptible to large deformation caused by shear and extrusion loads. Most of recent reports 
(a)

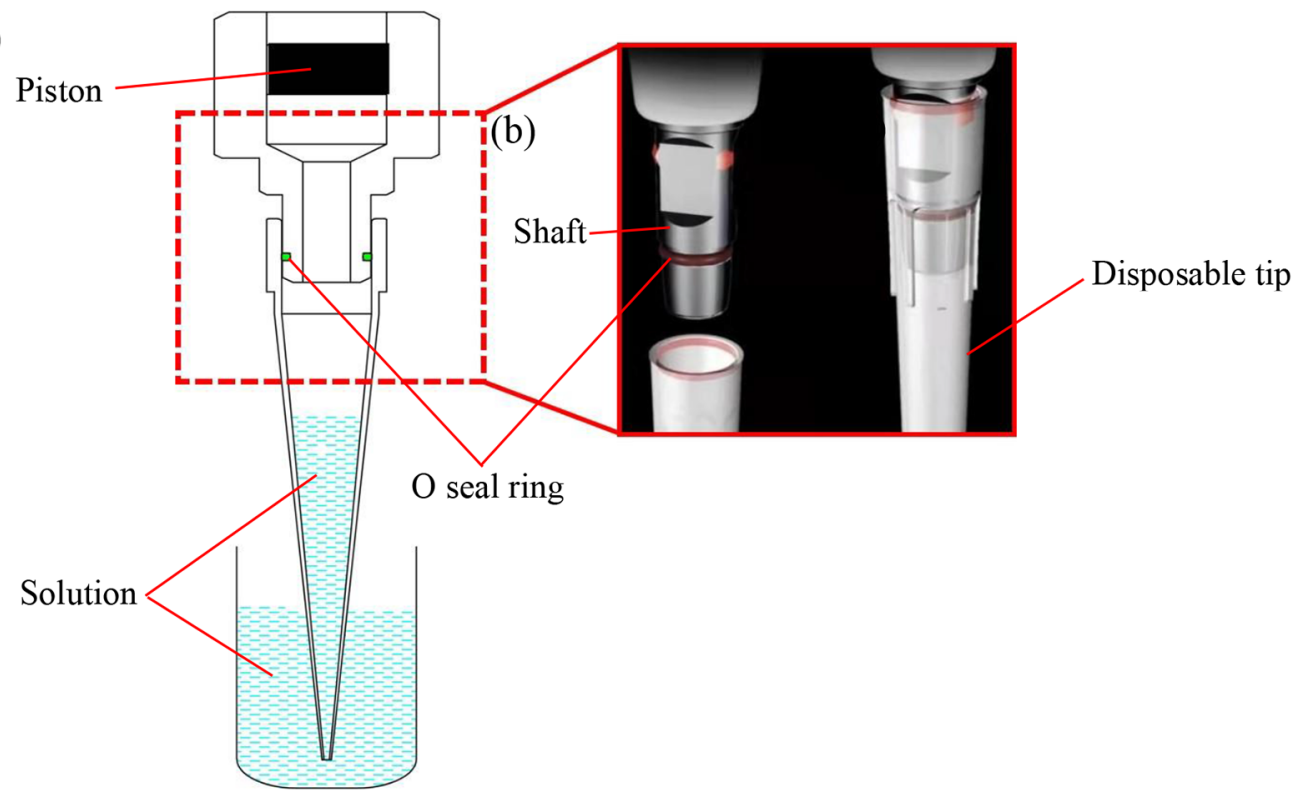

Fig. 1. The process of and pipette shaft and disposable tip assembly. (a) Diagrammatic figure of pipette device (b) Pipette shaft and disposable tip before assembly (c) Assembly diagram.

focus on the establishment of rubber fatigue life prediction models and fatigue life prediction. Griffith [1] proposed a crack extension energy criterion based on fracture mechanics in 1920, and argued that there was defect distribution in any material, and that the greatest defect would be the first to cause some damage. Rivlin and Thomas [2] successfully applied it to rubber tearing and proposed the concept of strain energy release rate. Zhang et al. [3,4] analyzed the impact of rubber and its geometry on the sealing performance of packer rubber. Also, they built a finite element model of four packer rubber materials using ABAQUS and FE-SAFE, and analyzed the effect of each material on sealing performance and fatigue life of the packer rubber. Ding et al. [5] built a rubber fatigue life prediction model based on the cumulative fatigue damage theory with the tearing energy range as a damage parameter. Wang et al. [6] studied the fatigue life of natural rubber under cyclic loading of tensile strain amplitude only according to the continuum damage mechanics principle. Tomita et al. [7] proposed an elastomer damage evolution model and life prediction method based on the damage theory. Wang and Wei [8] calculated the fatigue life of rubber materials in a rolling tire model by the critical plane method based on the rubber fatigue crack propagation theory. Luo et al. [9-11], considering the stress on a rubber damping spring in actual use, proposed to use an effective stress represented by three main stress values as a parameter for predicting the fatigue failure life. Also, they identified the fatigue life cycle corresponding to the maximum effective stress value according to the S-N curve of rubber materials, and adopted it as a basis for designing the fatigue life of rubber springs. In addition, some scholars analyze the effects of material laws, load conditions and reunion distribution on elastomer life prediction. For example, Yaagoubi et al.
[12-14], according to the particle distribution and crack expansion characteristics in the sample, propose a method for predicting the life of filled elastomers based on particle distribution and J-integral evaluation. Furthermore, Yaagoubi et al. [15-18] assessed tear energy through pants samples and predicted tear energy in pattern III for filled elastomers. The Ogden model is also used to predict the rate of energy release of filled elastomers and unfilled elastomers in crack open mode. It is noted that J-integral evaluation, taking into account stress softening, could be a promising method for estimating crack expansion or predicting life.

The above-mentioned literature studies mentioned various methods for predicting the fatigue life of rubber and various factors that affect the life prediction of elastomers. However, the fatigue life of rubber materials calculated by different fatigue damage parameters is different. Due to many factors affecting rubber fatigue, in the field of rubber fatigue analysis, there is no consensus on what damage parameters can predict the fatigue characteristics of rubber materials. So far, there has been no report on the analysis of the assembly process of the pipette sleeve seal ring and the prediction and research on the fatigue life of the seal ring under frequent assembly conditions. In view of the long period and high cost of model experiment under actual physical conditions, this study is based on the theory of continuum damage mechanics, combined with the special working conditions of frequent assembly of sleeve seals, and established the equivalent effect of sleeve seals in the assembly process. The fatigue life prediction model with the variable range as the damage parameter is used to calculate the fatigue life of the dangerous section element on the sleeve sealing ring. This research carried out an accurate life prediction for the pipette sleeve sealing ring, provided technical guarantee for 


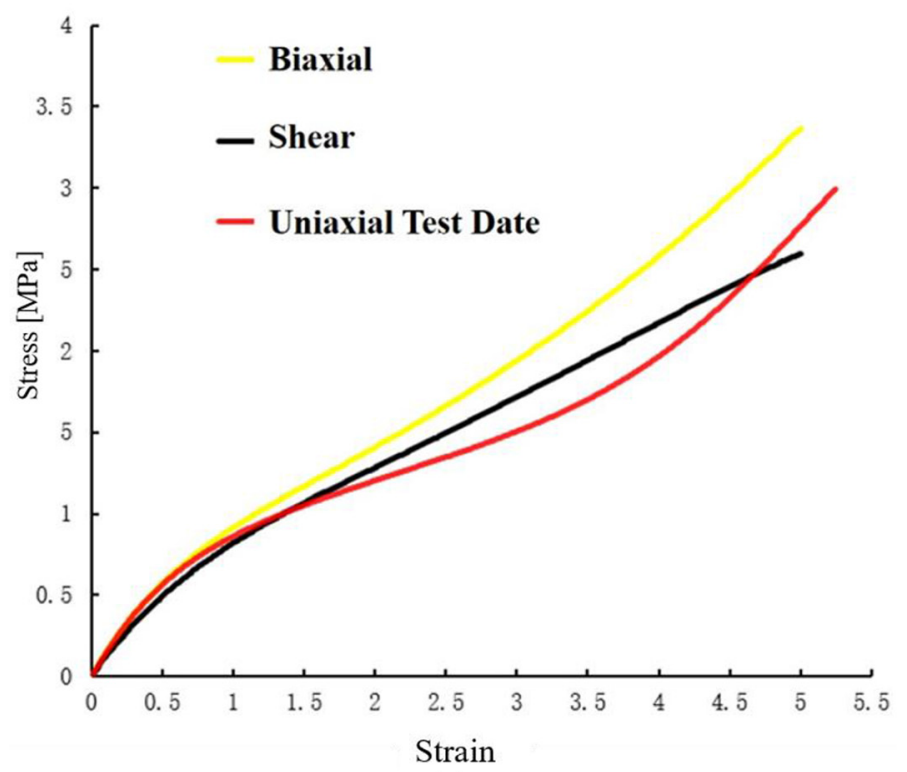

(a)

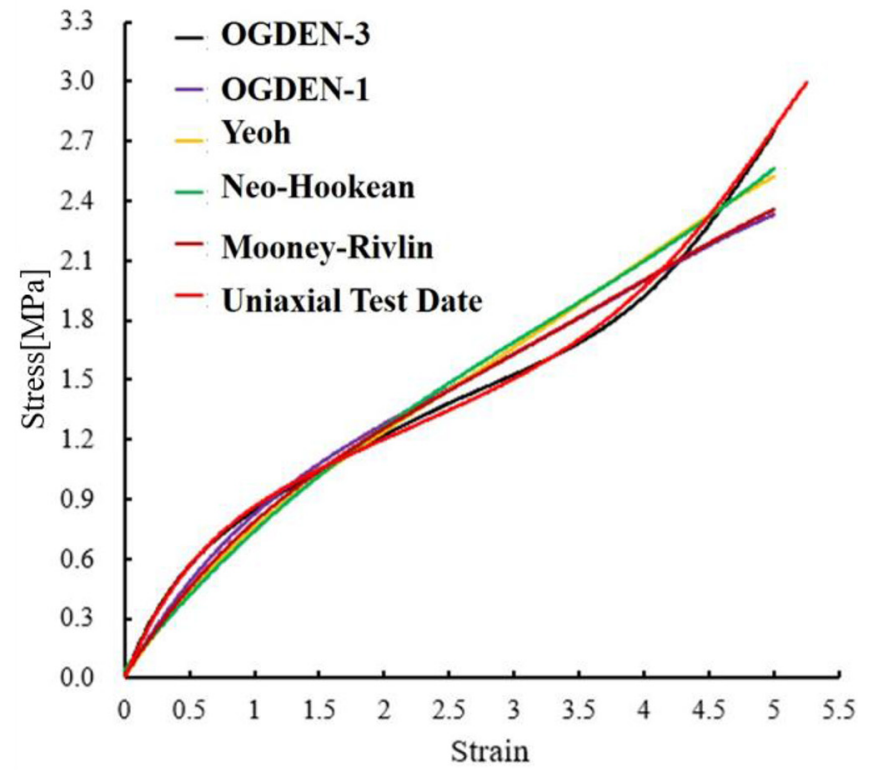

(b)

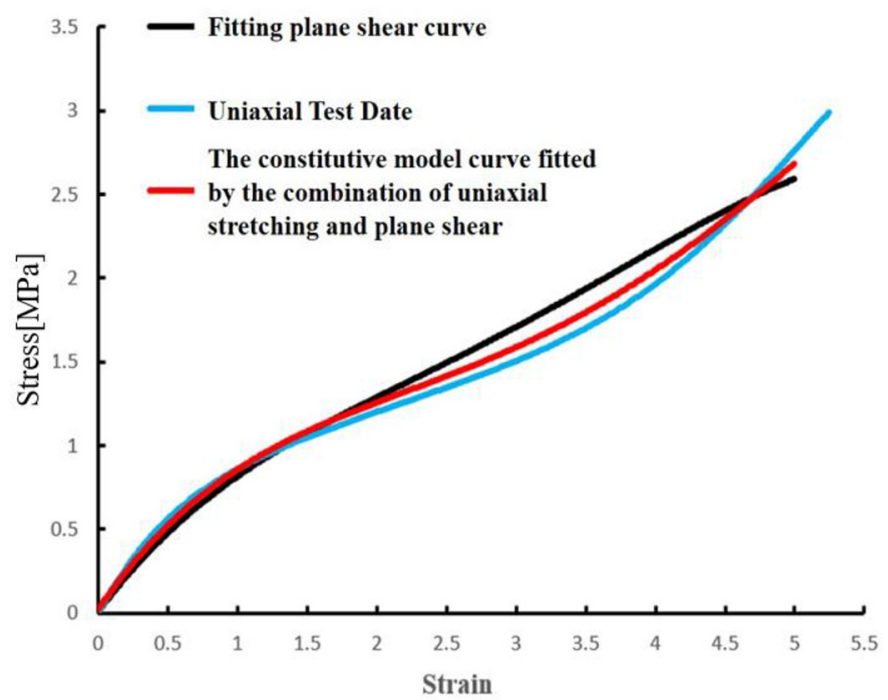

(c)

Fig. 2. Fitting curve of fatigue test and simulation. (a) Stress-strain curve of uniaxial tensile test. (b) Fitting curves of different rubber constitutive models. (c) Fitting curve of the combination of uniaxial tensile and plane shear.

the accuracy of pipette nucleic acid extraction, and provided theoretical guidance and design basis for the structure optimization of the pipette.

\section{Finite element analysis of seal ring and tip assembly}

Figure 3 shows the process of insertion of a shaft seal ring into a tip simulated in ABAQUS (see Fig. 3a for the assembly structure chart). When the rubber seal ring mounted on the pipette sleeve is matched with the pipette tip, its elastic modulus is much lower than the elastic modulus of the sleeve and the tip, so this article sets the sleeve and the tip as an analytical rigid body. Among them, the number of grid cells of the sleeve seal ring is 3653, using the $\mathrm{C} 3 \mathrm{D} 8 \mathrm{H}$ cell type, and the cell size (mesh size) is about $0.0083 \mathrm{~mm}$.

After comprehensively analyzing the advantages and disadvantages of several hyperelastic models, this study draws the following conclusions: the applicable range of Neo Hookean model is too narrow; the strain energy function of Yeoh model is inaccurate at small deformations and cannot explain isobiaxial tension well. Tensile experiment; Amuda-Boyce strain energy function has a complex form and is not suitable for biaxial tensile 
Table 1. Material parameters of the third-order Ogden model after fitting.

\begin{tabular}{llllll}
\hline$\mu_{1} / M P a$ & $\alpha_{1}$ & $\mu_{2} / M P a$ & $\alpha_{2}$ & $\mu_{3} / M P a$ & $\alpha_{3}$ \\
\hline 0.924 & 0.929 & -0.0143 & -0.593 & 0.0421 & 3.157 \\
\hline
\end{tabular}

experiment; Mooney-Rivilin model is currently the most commonly used rubber material model due to its simple model and easy determination of parameters, but this model is mainly applicable In the case of relatively small deformation of rubber materials, it is impossible to make a reasonable explanation for the mechanical behavior of large deformation of rubber materials. Compared with the Ogden model, the Mooney-Rivlin model has no obvious advantages, and the Ogden model is more concise in form than the Mooney-Rivilin model, and is used in uniaxial stretching, pure shear, and isobiaxial stretching experiments. All have been verified $[19,20]$. Therefore, in this simulation, the Ogden model was selected as the hyperelastic model.

Yanshan et al. [21] pointed out that when only uniaxial tensile test data is available, finite element simulation can be used to obtain isobiaxial tensile and plane shear data, and then use different combinations between them. Fit material parameters. The sleeve seal ring in this study has a relatively large strain during the insertion of the tip. After comparing various combinations, it is found that the material parameters obtained by the combination of uniaxial stretching and plane shear of the Ogden model are able to obtain higher accuracy. In this study, the stressstrain curve obtained by uniaxial tensile test is shown in Figure 2a. For different rubber constitutive models, the fitting curve is shown in Figure 2b. The fitting curve of constitutive parameters obtained by the combination of uniaxial stretching and plane shearing is shown in Figure 2c. And finally, the material parameters of the Ogden model was obtained by regression analysis and data processing, as shown in Table 1. Then the fitting results are applied to the large deformation contact analysis of the sleeve seal ring, which provides a reference for the prediction of the fatigue life of the seal ring later.

The simulation results as shown: In the assembly process, the seal ring comes in contact with a side of the seal groove first. At this time, the seal ring deforms slightly, and therefore doesn't generate a high shear stress, as shown in Figure 3b. Subsequently, the seal ring starts touching the tip fillet $\mathrm{R}$ with very low stress at this time, as shown in Figure 3c. Assembly continues, under the squeezing action of the tip fillet, the shear stress of the seal ring increases gradually. At this time, the seal ring will easily suffer shear failure, and finally, a maximum shear stress appears, as shown in Figure 3d. When the seal ring starts sliding into the inner cavity of the tip, the shear stress decreases gradually, as shown in Figure 3e. After the entry of the entire seal ring into the inner cavity of the tip, the seal ring primarily comes under a compressive stress, as shown in Figure 3f.

As can be seen in Figure 3d, whenever it is inserted into the tip, the seal ring will bear a high shear stress here, and the tip, a disposable component, will be removed and changed for new after each pipetting operation. Therefore, the seal ring will have to come under a shear stress and compressive stress for heaps of times, so it easily suffers shear failure, thereby affecting the airtightness of the pipette.

\section{Fatigue prediction and analysis of shaft seal rings}

\subsection{Fatigue life model of shaft seal rings}

An O-ring is supposed to have high elasticity and resilience, proper mechanical strength, stable performance, and noncorrosiveness. The O-ring researched in this study is made of rubber. In this paper, based on the continuum damage mechanics theory, the first-order Ogden strain energy function and unit-volume elastic strain energy function are used to describe the characteristics of rubber. The strain energy of rubber in the uniaxial stress state can be expressed by equation (1), while the relationship between stress and strain can be expressed by equation (2) [2].

$$
\begin{gathered}
U=\frac{2 \mu_{1}}{\alpha_{1}^{2}} \cdot\left(\lambda_{U}^{\alpha_{1}}+2 \lambda_{U}^{-\alpha_{1} / 2}-3\right) \\
=\frac{2 \mu_{1}}{\alpha_{1}^{2}} \cdot\left[\left(1+\varepsilon_{U}\right)^{\alpha_{1}}+2\left(1+\varepsilon_{U}\right)^{-\alpha_{1} / 2}-3\right] \\
\sigma_{U}=\frac{\partial U}{\partial \lambda_{U}}=\frac{2 \mu_{1}}{\alpha_{1}}\left[\left(1+\varepsilon_{U}\right)^{\alpha_{1}-1}-\left(1+\varepsilon_{U}\right)^{-\left(\alpha_{1} / 2+1\right)}\right]
\end{gathered}
$$

where $\alpha_{1}$ and $\mu_{1}$ are constitutive parameters. The stressstrain data obtained in the uniaxial tensile test can be fitted to get the following result: $\mu_{1}=1.21[\mathrm{MPa}], \alpha_{1}=1.956 ; \lambda_{U}$ represents the principal elongation ratio in the unilateral stretching state; $\varepsilon_{U}$ is the strain in the unilateral stretching state; $\sigma_{U}$ represents the stress in unilateral stretching state.

The fatigue wear failure of rubber caused under the action of a cyclic fatigue load can be described by defining a damage factor $D$, as shown in equation (3), while fatigue damage in a single cycle can be expressed by equation (4) [14].

$$
\begin{gathered}
\bar{\sigma}=\sigma_{U} /(1-D) \\
\frac{d D}{d N}=\left\{\frac{2 \mu_{1}}{\alpha_{1} \cdot b} \times \frac{\left[\Delta \lambda_{U}^{\alpha_{1}-1}-\Delta \lambda_{U}^{-\left(\alpha_{1} / 2+1\right)}\right]^{2}}{\left[\left(\alpha_{1}-1\right) \cdot \Delta \lambda_{U}^{\alpha_{1}-2}+\left(\alpha_{1} / 2+1\right) \cdot \Delta \lambda_{U}^{-\left(\alpha_{1} / 2+1\right)}\right]}\right\}^{a}
\end{gathered}
$$

where $\bar{\sigma}$ represents a damaging stress; $\mathrm{N}$ represents a cycle; $\Delta \lambda_{U}$ represents principal elongation ratio range. 

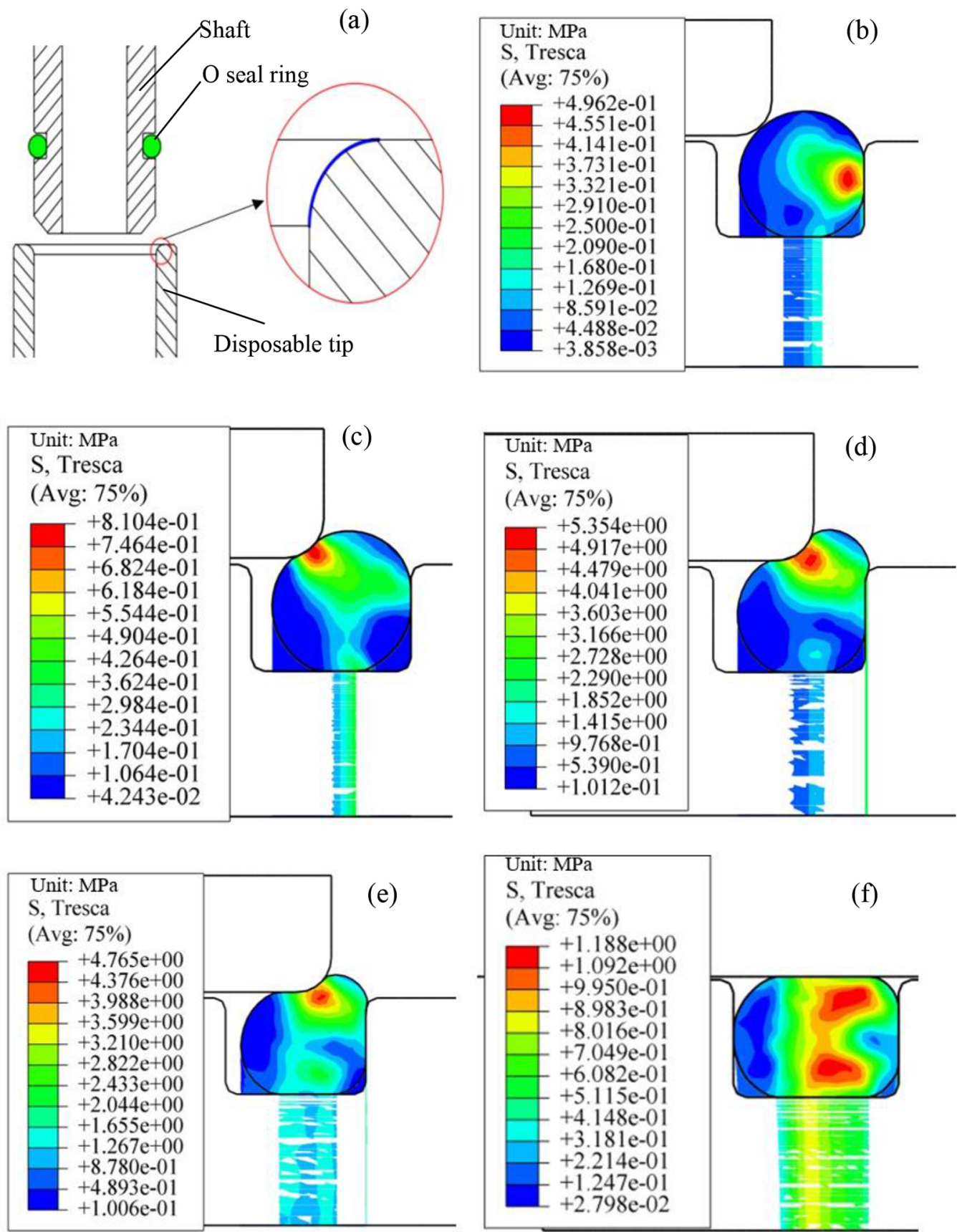

Fig. 3. The whole assembly process of insertion of a seal ring into the disposable tip. (a) Assembly structure drawing. (b) The seal ring is in contact with a side of the seal groove. (c) The seal ring is in initial contact with the tip fillet R. (d) The seal ring is in complete contact with the tip fillet R. (e) The seal ring starts sliding into the inner cavity of the tip. (f) The seal ring has completely slid into the inner cavity of the tip till installation finish.

As can be seen in Figure 3, the shaft seal ring will suffer large extrusion deformation when inserted into the tip. Owing to the non-linearity of rubber, when the strain of the seal ring is high, the accuracy of the magnitude and distribution of its stress will decrease. Therefore, the use of an effective strain range as the fatigue failure parameter is more in line with the actual fatigue life of the shaft seal ring.

$$
\begin{aligned}
& N_{f}=(1+2 a)^{-1} \\
& \left\{\frac{2 \mu_{1}}{\alpha_{1} \cdot b} \times \frac{\left[\left(1+\Delta \varepsilon_{U}\right)^{\alpha_{1}-1}-\left(1+\Delta \varepsilon_{U}\right)^{-\left(\alpha_{1} / 2+1\right)}\right]^{2}}{\left(\alpha_{1}-1\right)\left(1+\Delta \varepsilon_{U}\right)^{\alpha_{1}-2}+\left(\alpha_{1} / 2+1\right)\left(1+\Delta \varepsilon_{U}\right)^{-\left(\alpha_{1} / 2+2\right)}}\right\}^{-a}
\end{aligned}
$$


Table 2. Compound formula of pipette shaft seal ring.

\begin{tabular}{llllllll}
\hline Rubber material & NBR & Zinc oxide & $\begin{array}{l}\text { stearic } \\
\text { acid }\end{array}$ & $\begin{array}{l}\text { Antioxidant } \\
\text { RD }\end{array}$ & $\begin{array}{l}\text { Antioxidant } \\
\text { MB }\end{array}$ & $\begin{array}{l}\text { Spray carbon } \\
\text { black }\end{array}$ & HAF \\
\hline PHR & 100 & 5 & 1 & 1 & 1 & 40 & 55 \\
\hline Rubber material & DBP & Dibutyl sebacate & DCP & Accelerator CZ & Accelerator M & Sulphur & Total \\
\hline PHR & 10 & 15 & 2 & 1.5 & 0.5 & 0.3 & 232.3 \\
\hline
\end{tabular}

NBR: Nitrile Butadiene Rubber. HAF: High abrasion furnace black. DBP: dibutyl phthalate. DCP: Dicumylperoxidecas.

where $N_{f}$ represents the fatigue life of the shaft seal ring; $\Delta \varepsilon_{U}$ represents effective strain; a and be are material constants, of which $a=2, b=2163$ [15].

Aidy et al. [22,23] conducted a uniaxial tensile fatigue test on dumbbell-shaped rubber samples with a Shore hardness of about 60 , using sine wave displacement control, and the test frequency was $0.1 \mathrm{~Hz}$. substituting the test data into equation (5) to obtain the material constant $a=2$ and $b=2.163$. Since the rubber material of the sleeve sealing ring in this paper is also about 60 Shore hardness, and its rubber formula is shown in Table 2, this study continues to use this data for subsequent fatigue life analysis.

\subsection{Equivalent strain calculation formula}

As can be seen in equation (5), effective strain will be adopted in this paper to calculate the fatigue life of the shaft seal ring in complex stress state. The effective strain will be calculated using equation (6)

$$
\varepsilon_{f}=\sqrt{\varepsilon_{1}^{2}+A \varepsilon_{2}^{2}+B \varepsilon_{3}^{2}}
$$

where $\varepsilon_{1}, \varepsilon_{2}$ and $\varepsilon_{3}$ represent the principal strain in three directions, respectively; $\varepsilon_{f}$ represents effective strain. They are in line with the following hypothesis:

$-\varepsilon_{1}>0, \varepsilon_{1} \geq \varepsilon_{2} \geq \varepsilon_{3}$

$-A=\left\{\begin{array}{ll}0 & \varepsilon_{2} \leq 0 \\ 1 & \varepsilon_{2}>0\end{array}, B=\left\{\begin{array}{ll}0 & \varepsilon_{3} \leq 0 \\ 1 & \varepsilon_{3}>0\end{array}\right.\right.$.

\subsection{Calculation of the fatigue life of shaft seal rings}

\subsubsection{Calculation of effective strain range for dangerous} cross-section

The initiation and propagation of fatigue cracks in rubber depend on the magnitude of its maximum principal stress or maximum principal strain. So, crack propagation in a rubber seal ring can be identified according to the first principal strain in one cycle. Figure 4 shows the changes in the nominal strain borne by the dangerous cross-section segments on the seal ring in a one-cycle assembly process of a disposable pipette tip. The distribution of principal strain in the shaft seal ring is detected based on a finite-element analysis, preliminarily identifying potential crack areas, as

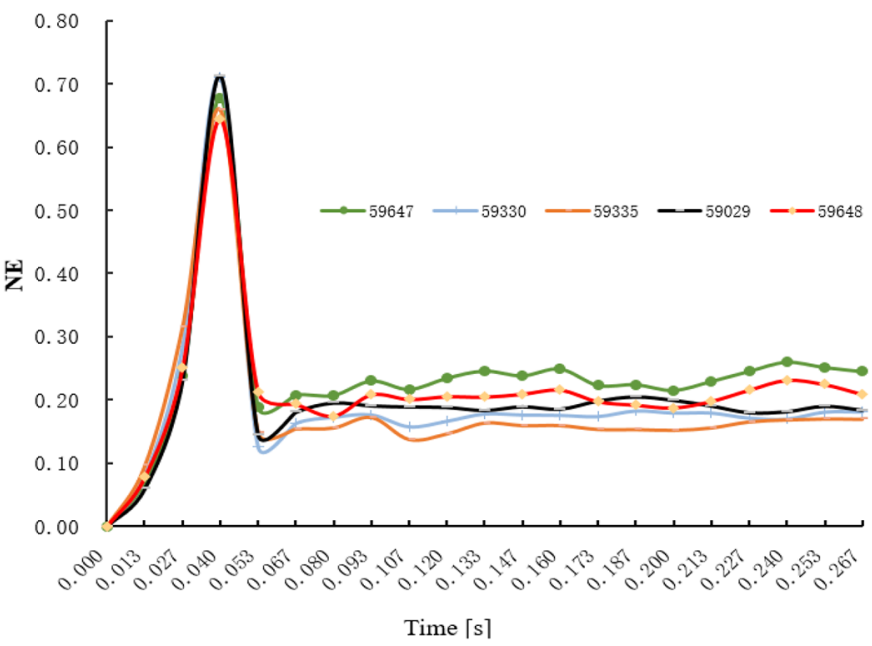

Fig. 4. The change of nominal strain of the dangerous section of the sleeve seal ring during the assembly process.

shown in Figure 5. According to the condition of the first principal strain in equation (6), three principal strains are certain dangerous cross-section segments of the shaft seal ring can be extracted during its frequent insertion into tips. The effective strains obtained according to equation (6) are shown in Tables 3-5.

According to the fatigue damage theory and equation (5), the fatigue damage parameter for fatigue prediction should be an effective strain range rather than a maximum effective strain. An effective strain range of the dangerous cross-section segments on the seal ring can be obtained from Tables 3-5, as shown in Table 6 .

\subsubsection{Fatigue life calculation and analysis}

The effective strain range value in Table 6 is substituted into equation (5), finally figuring out the fatigue crack initiation life of the dangerous cross-section segments of the shaft seal ring, as shown in Table 7 . The minimum number of fatigue life cycles is $9.862 \mathrm{E} 5$, and the corresponding Segment No. is 59029. Worn-out pipette shaft real rings are investigated, showing that most fatigue cracks are initiated in the surface layer of the seal rings and then propagated, as shown in Figure 6. As shown in the figure, the wear locations on shaft real rings generated in actual use are the same as the sites of the dangerous cross-section segments identified by finite element simulation. 


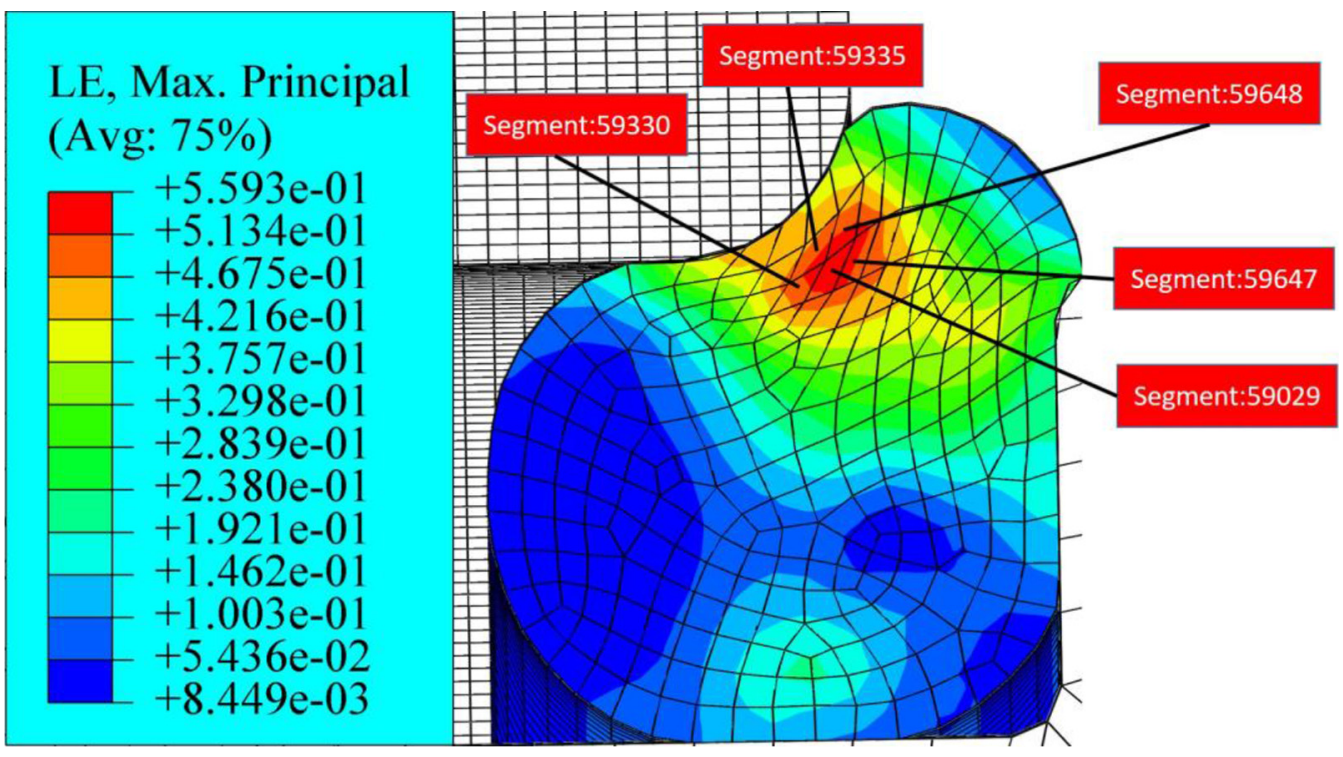

Fig. 5. Distribution of dangerous cross-section segments on the seal ring during shaft-disposable tip assembly.

Table 3. Effective strain of the dangerous cross-section segments on the seal ring during fillet contact.

\begin{tabular}{lllll}
\hline Segment no. & $\varepsilon_{1}$ & $\varepsilon_{2}$ & $\varepsilon_{3}$ & $\varepsilon_{f 1}$ \\
\hline 59330 & 0.5485 & $3.886 \times 10^{-3}$ & -0.5809 & 0.5485 \\
59029 & 0.5593 & $5.261 \times 10^{-3}$ & -0.5735 & 0.5593 \\
59335 & 0.5512 & $4.263 \times 10^{-3}$ & -0.5910 & 0.5512 \\
59647 & 0.5341 & $4.588 \times 10^{-3}$ & -0.5858 & 0.5341 \\
59648 & 0.5315 & $5.826 \times 10^{-3}$ & -0.5625 & 0.5315 \\
\hline
\end{tabular}

Table 4. Effective strain of the dangerous cross-section segments on the seal ring in the inner cavity of the tip.

\begin{tabular}{lllll}
\hline Segment no. & $\varepsilon_{1}$ & $\varepsilon_{2}$ & $\varepsilon_{3}$ & $\varepsilon_{f 2}$ \\
\hline 59330 & 0.2273 & -0.0295 & -0.2375 & 0.2273 \\
59029 & 0.2470 & -0.0340 & -0.2542 & 0.2470 \\
59335 & 0.2610 & -0.0315 & -0.2660 & 0.2610 \\
59647 & 0.2324 & -0.0335 & -0.2433 & 0.2324 \\
59648 & 0.2195 & -0.0357 & -0.2659 & 0.2195 \\
\hline
\end{tabular}

Table 5. Effective strain of the dangerous cross-section segments on the seal ring in natural state.

\begin{tabular}{lllll}
\hline Segment no. & $\varepsilon_{1}$ & $\varepsilon_{2}$ & $\varepsilon_{3}$ & $\varepsilon_{f 3}$ \\
\hline 59330 & $2.605 \times 10^{-2}$ & $6.05 \times 10^{-3}$ & $-16.20 \times 10^{-3}$ & $2.651 \times 10^{-3}$ \\
59029 & $2.055 \times 10^{-2}$ & $4.80 \times 10^{-3}$ & $-51.50 \times 10^{-3}$ & $2.154 \times 10^{-3}$ \\
59335 & $1.518 \times 10^{-2}$ & $-2.05 \times 10^{-3}$ & $-31.00 \times 10^{-3}$ & $1.518 \times 10^{-3}$ \\
59647 & $1.785 \times 10^{-2}$ & $1.35 \times 10^{-3}$ & $-41.20 \times 10^{-3}$ & $1.792 \times 10^{-3}$ \\
59648 & $2.892 \times 10^{-2}$ & $0.75 \times 10^{-3}$ & $-39.15 \times 10^{-3}$ & $2.893 \times 10^{-3}$ \\
\hline
\end{tabular}


Table 6. Effective strain range of the dangerous area of the seal ring.

\begin{tabular}{llllll}
\hline Segment no. & $\varepsilon_{f 1}$ & $\varepsilon_{f 2}$ & $\varepsilon_{f 3}$ & $\Delta \varepsilon_{f 1-2}=\varepsilon_{f 1}-\varepsilon_{f 2}$ & $\Delta \varepsilon_{f 1-3}=\varepsilon_{f 1}-\varepsilon_{f 3}$ \\
\hline 59330 & 0.5485 & 0.2273 & $2.651 \times 10^{-2}$ & 0.3212 & 0.5220 \\
59029 & 0.5593 & 0.2470 & $2.154 \times 10^{-2}$ & 0.3123 & 0.5378 \\
59335 & 0.5512 & 0.2610 & $1.518 \times 10^{-2}$ & 0.2902 & 0.5361 \\
59647 & 0.5341 & 0.2324 & $1.792 \times 10^{-2}$ & 0.3017 & 0.5162 \\
59648 & 0.5315 & 0.2195 & $2.893 \times 10^{-2}$ & 0.3120 & 0.5026 \\
\hline
\end{tabular}

Table 7. Prediction of the fatigue life of shaft seal rings.

\begin{tabular}{lrr}
\hline Segment no. & \multicolumn{1}{c}{$N_{f 1-3}$} & $N_{f 1-2}$ \\
\hline 59330 & 1102836.10 & 7081079.03 \\
59029 & 986145.69 & 7899367.22 \\
59335 & 997909.65 & 10517979.44 \\
59647 & 1151805.54 & 9037368.09 \\
59648 & 1271743.53 & 7928988.92 \\
\hline
\end{tabular}

(a)

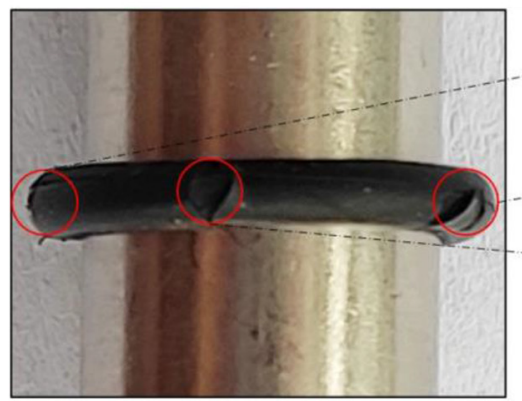

(b)

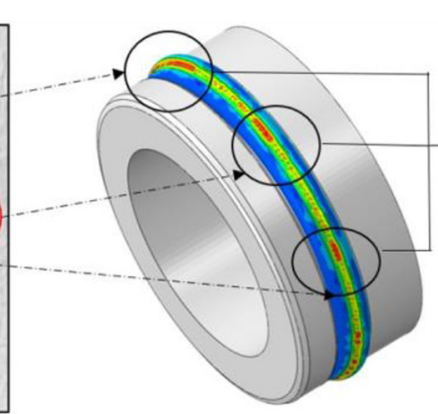

Unit: MPa $\mathrm{S}$, Tresca (Avg: $75 \%$

$+5.354 \mathrm{e}+00$

(c) $+4.479 \mathrm{e}+00$
$+4.041 \mathrm{e}+00$ $+4.04 \mathrm{e}+00$
$-+3.603 \mathrm{e}+00$
$+3.166 \mathrm{e}+00$ $-+3.166 \mathrm{e}+0$ $+3.166 \mathrm{e}+00$
$+2.728 \mathrm{e}+00$
$++2.290 \mathrm{e}+00$
$+1.852 \mathrm{e}+00$ $+1.852 \mathrm{e}+00$
$+1.45 \mathrm{e}+00$ $+1.458 \mathrm{e}-01$
++3901 $\begin{array}{r}+5.390 \mathrm{e}-01 \\ +1.012 \mathrm{e}-01 \\ \hline\end{array}$

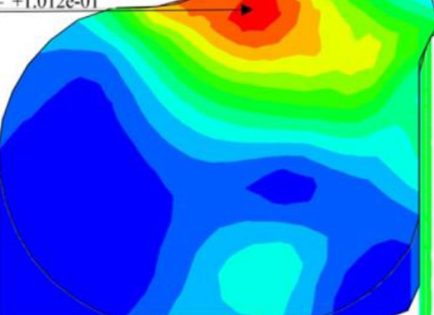

Fig. 6. The physical picture of the damaged shaft seal ring and the shear stress nephogram of the dangerous section. (a) Failed shaft seal ring (b) Simulation shear stress nephogram of assembly process (c) Profile of dangerous section of shaft seal ring.

In summary, during the insertion of a shaft seal ring into tips, the seal ring's contact with the tip fillet is the reason for an increase in the shear stress of the seal ring. Thus, it can be seen that the inner angle of tips should be arc-shaped because a transition arc can effectively decrease the shear stress peak. For the purchase of disposable tips, it is suggested to select tips with a long fillet radius, which can not only enable a shaft seal ring to slide into the inner cavity of tips easily, but prolong the service life of the seal ring.

\section{Conclusions}

- Based on the test data of uniaxial tension, the data of equi-biaxial tension and plane shear are solved by simulation, and the material parameters of the Ogden model are determined.

- The assembly process of the sleeve sealing ring and disposable tip is simulated, and the dangerous section position of the sealing ring is judged.

- Based on the theory of continuum damage mechanics, a fatigue life prediction model with the equivalent strain range as the damage parameter is established and the fatigue life of the dangerous section of the sleeve seal ring is calculated.

- According to the comparison of actual survey results, it is found that the location of the sleeve seal ring wear and damage is basically the same as the position of the dangerous section unit calculated in this paper, and the number of uses of the sleeve seal ring before wear is 
basically the same as the lowest life of the dangerous unit. With the same value, the error of predicting fatigue life is less than $20 \%$.

- Through analysis, it is found that the inner rounded corner of the tip is the cause of the increase in the shear stress of the sealing ring, so the inner corner of the tip should be set in an arc shape.

\section{Declaration of conflicting interests}

The author(s) declared no potential conflicts of interest with respect to the research, authorship, and/or publication of this article.

This study is supported by Key project of Guangxi province, PR of China (Grant Number: 2021KY1069).

\section{Nomenclatures}

$\varepsilon_{1} \quad$ Principal strain 1

$\varepsilon_{2} \quad$ Principal strain 2

$\varepsilon_{3} \quad$ Principal strain 3

$\varepsilon_{f 1} \quad$ Effective strain 1

$\varepsilon_{f 2} \quad$ Effective strain 2

$\varepsilon_{f 3} \quad$ Effective strain 3

$\Delta \varepsilon_{f 1-2}$ Effective strain range (before assembly-the seal ring is in complete contact with the tip fillet $\mathrm{R}$ )

$\Delta \varepsilon_{f 1-3}$ Effective strain range (before assembly- the seal ring has completely slid into the inner cavity of the tip)

$N_{f 1-3} \quad$ Fatigue life of shaft seal ring (before assemblycontacting with the tip fillet $\mathrm{R}$ )

$N_{f 1-2} \quad$ Fatigue life of the seal ring (contacting with the tip fillet $\mathrm{R}$ - sliding into the inner cavity of the tip)

\section{References}

[1] A.A. Griffith, The phenomena of rupture and flow in solids, Philos. Trans. Royal Soc. Lond. Ser A 221, 582-593 (1921)

[2] R.S. Rivlin, A.G. Thomas, Rupture of rubber. I. Characteristic energy for tearing, J. Polym. Sci. 10, 291-318 (1953)

[3] F. Zhang, X. Jiang et al., Mechanical analysis of sealing performance for compression packer rubber tube, Mech. Ind. 19, 309 (2018)

[4] F. Zhang, H. Shui, J.-M. Yang, Sealing performance and fatigue life of the fracturing packer rubber of various materials, Mech. Eng. Sci. 233, 6157-6166 (2019)

[5] D. Zhiping, C. Jieping, S. Chuanjiang, Analysis of fatigue crack growth life for rubber vibration damper, J. Mech. Eng. 046, 58-64 (2010)

[6] B. Wang, H. Lu, G.H. Kim, A damage model for the fatigue life of elastomeric materials, Mech. Mater. 34, 475-483 (2002)
[7] Y. Tomita, W. Lu, Computational characterization of microto macroscopic mechanical behavior and damage of polymers containing second-phase particles, Int. J. Damage Mech. 11, 129-149 (2002)

[8] W. Hao, W. Yingtao, Prediction on rubber fatigue life of tire based on fatigue crack propagation theory, Tire Ind. 36, 259-266 (2016)

[9] R.K. Luo, P.W. Cook, W.X. Wu et al., Fatigue design of rubber springs used in rail vehicle suspensions, Proc. Instn Mech. Eng. F 217, 237-240 (2003)

[10] R.K. Luo, P.W. Cook, W.X. Wu et al., An approach to evaluate the service life of rubber springs used in rail vehicle suspensions, Proc. Instn Mech. Eng. Part F 218, 173-177 (2004)

[11] R.K. Luo, W.X. Wu, Fatigue failure analysis of antivibration rubber spring, Eng. Fail. Anal. 13, 110-116 (2006)

[12] M. El Yaagoubi, D. Juhre, J. Meier, N. Kröger, T. Alshuth, U. Giese, Lifetime prediction of filled elastomers based on particle distribution and the J-integral evaluation, Int. J. Fatigue 112, 341-354 (2018)

[13] O. Gehrmann, M. El Yaagoubi, H. El Maanaoui, J. Meier, Lifetime prediction of simple shear loaded filled elastomers based on the probability distribution of particles, Polym. Test. 75, 229-236 (2019)

[14] E.Y. Mohammed, M. Jens, J. Daniel, Lifetime prediction of carbon black filled elastomers based on the probability distribution of particle using an inelastic and hyperelastic material model, Eng. Fail. Anal. 118 (2020)

[15] M.E. Yaagoubi, H.E. Maanaoui, J. Meier, New fatigue test sample: Lifetime prediction of carbon black filled elastomers based on the probability distribution of particles, Polymer $208(2020)$

[16] M. El Yaagoubi, D. Juhre, J. Meier, T. Alshuth, U. Giese, Prediction of tearing energy in mode III for filled elastomers, Theor. Appl. Fract. Mech. 88, 31-38 (2016)

[17] M. El Yaagoubi, D. Juhre, J. Meier, T. Alshuth, U. Giese, Prediction of energy release rate in crack opening mode (mode I) for filled and unfilled elastomers using the Ogden model, Eng. Fract. Mech. 182, 74-85 (2017)

[18] M. El Yaagoubi, D. Juhre, J. Meier, T. Alshuth, U. Giese, Tearing energy and path-dependent J-integral evaluation considering stress softening for carbon black reinforced elastomers, Eng. Fract. Mech. 190, 259-272 (2018)

[19] S. Jianbing, X. Sufang, L. Baohui, Large deformation contact analysis of O-type rubber seal ring based on Ogden model, Lubric. Eng. 39, 38-42 (2014)

[20] C. Jiazhao, H. Mingxiang, W. Xueren, W. Ting, Several typical constitutive models of rubber materials and their applicability, Mater. Rep. 29, 118-120+124 (2015)

[21] Y. Shan, W. Wei, Determination of material parameters in rubber-like superelastic constitutive model, China Rubber Ind. 61, 453-457 (2014)

[22] A. Aidy, M. Hosseini, S. Barkawi, Continuum damage mechanics modeling For fatigue life of elastomeric materials, Int. J. Struct. Integr. 1, 63-72 (2010)

[23] D. Zhiping, Y. Ronghua, H. Youjian, Fatigue life analysis of rubber elastic damping components based on continuous damage model, J. Mech. Eng. 50, 80-86 (2014)

Cite this article as: C. Wang, Q. Su, Z. Huang, Z. Lian, Y. Liu, Effective strain range-based prediction and analysis of the fatigue life of pipette shaft seal ring, Mechanics \& Industry 22, 40 (2021) 